

\section{The Paradox Between Resistance to Hypoxia and Liability to Hypoxic Damage in Hyperglycemic Peripheral Nerves Evidence for Glycolysis Involvement UWE SCHNEIDER, WOLFGANG NIEDERMEIER, AND PETER GRAFE}

\begin{abstract}
Isolated ventral and dorsal rat spinal roots incubated in normal $(2.5 \mathrm{mM})$ or high glucose $(25 \mathrm{mM})$ concentrations or in high concentrations of other hexoses were exposed transiently to hypoxia (30 min) in a solution of low buffering power. Compound nerve action potentials, extracellular direct current potentials, and interstitial pH were continuously recorded before, during, and after hypoxia. Ventral roots incubated in 25 $\mathrm{mM} \mathrm{D}$-glucose showed resistance to hypoxia. Dorsal roots, on the other hand, revealed electrophysiological damage by hyperglycemic hypoxia as indicated by a lack of posthypoxic recovery. In both types of spinal roots, interstitial acidification was most pronounced during hyperglycemic hypoxia. The changes in the sensitivity to hypoxia induced by high concentrations of $\mathrm{D}$-glucose were imitated by high concentrations of D-mannose. In contrast, D-galactose, L-glucose, D-fructose, and L-fucose did not have such effects. Resistance to hypoxia, hypoxia-generated interstitial acidification, and hypoxia-induced electrophysiological damage were absent after pharmacological inhibition of nerve glycolysis with iodoacetate. These observations indicate 1) that enhanced anaerobic glycolysis produces resistance to hypoxia in hyperglycemic peripheral nerves and 2) that acidification may impair the function of peripheral axons when anaerobic glycolysis proceeds in a tissue with reduced buffering power. Diabetes 42:981-87, 1993
\end{abstract}

\footnotetext{
From the Department of Physiology, University of Munich, Munich, Germany. Address correspondence and reprint requests to Dr. Peter Grafe, Department of Physiology, University of Munich, Pettenkoferstrasse 12, D-80336 Munich, Germany.

Received for publication 14 October 1992 and accepted in revised form 18 February 1993

$\mathrm{pO}_{2}$, oxygen partial pressure; CNAP, compound nerve action potential; $\mathrm{pH}_{\mathrm{e}}$, interstitial $\mathrm{pH}$.
}

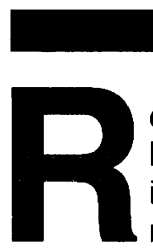

esistance to ischemia and liability to ischemic lesions are characteristics of peripheral nerves in diabetic patients $(1,2)$. However, the precise mechanisms underlying these alterations are not yet clear. Resistance to ischemia was first described by Steiness (3), and several hypotheses have attempted to explain this phenomenon (4): 1) a decrease in the accumulation of extracellular $\mathrm{K}^{+}$during ischemia caused by a change of a nodal diffusion barrier (5) or an enlarged interstitial endoneurial space $(6) ; 2)$ changes in the internodal length of myelinated axons (7); 3) increased availability of energy sources for anaerobic glycolysis in diabetic nerve $(8-12) ; 4)$ other alterations in neuronal metabolism such as inhibition of inositol uptake, accumulation of polyols, and/or reduced activity of the $\mathrm{Na}^{+} / \mathrm{K}^{+}$ATPase (13), and 5) adaptation to chronic hypoxemia (14-16).

The increased frequency of compression and entrapment neuropathies (17), such as carpal tunnel syndrome (18), indicates a liability to ischemic lesions in diabetic patients. Another example of this phenomenon is the observation that ischemia aggravates neuropathy in diabetic humans (19) and rats (20). The pathophysiology of liability to ischemic lesions is even less understood than the one underlying resistance to ischemia. Low (1) discussed the possibility that fiber degeneration may result from a fall of blood flow to a level of hypoxia that is no longer compensated by resistance to ischemia. Nukada (20) concluded that chronic endoneurial hypoxia is likely to cause a lowered threshold to ischemic tolerance in diabetic nerve.

We thought it important to clarify the mechanism of the abnormal sensitivity to hypoxia in hyperglycemic nerves, because this may help to gain insight into the pathogenesis of diabetic neuropathies. Resistance to hypoxia and liability to hypoxic damage have also been observed in isolated frog $(21)$ and rat nerves $(22,23)$ exposed to 
hypoxia in bathing solutions with high glucose concentrations. Thereby, for reasons we do not know, resistance to hypoxia was revealed clearly in motor fibers of ventral spinal roots, whereas sensory fibers in dorsal spinal roots were more liable to hypoxia-induced electrophysiological damage (23). Isolated nerves offer methodological advantages compared with animal experiments. For example, isolated nerves make it possible to alter the availability of substrates during exposure of the nerves to hypoxia. Also, neuronal metabolism can be modified without the need to consider possible changes in blood flow. In this study, we used these advantages to explore the experimental questions raised. First, exposure to hypoxia was performed in the presence of $D$-glucose or of high concentrations of other hexoses (L-glucose, D-fructose, D-galactose, D-mannose, and L-fucose). Second, effects of hypoxia on spinal roots were explored during inhibition of glycolysis. These experiments provide strong evidence for the involvement of glycolysis in both resistance to hypoxia and liability to hypoxia-related electrophysiological damage of hyperglycemic peripheral nerves.

\section{RESEARCH DESIGN AND METHODS}

Male Wistar rats, weighing 300-400 g, were obtained from Thomae (Biberach, Germany). The animals were anesthetized with urethan $(1.5 \mathrm{~g} / \mathrm{kg}$, intraperitoneally, supplemented as required) for a laminectomy to expose the cauda equina and the spinal ganglia. Spinal roots were removed in their entire length (from the spinal cord to the spinal nerve) for in vitro recording. The anatomical relationship of the isolated roots to the spinal ganglia enabled us to differentiate between dorsal and ventral roots. After preparation and before transfer to the experimental organ bath, the spinal roots were preincubated at room temperature in solutions with different hexoses. Previously, we have shown that a preincubation period of $30 \mathrm{~min}$ to $\sim 6 \mathrm{~h}$ in $2.5 \mathrm{mM} \mathrm{D}$-glucose resulted in a uniform response to hypoxia. The altered sensitivity to hypoxia in high concentrations of D-glucose, on the other hand, required at least $2 \mathrm{~h}$ of preincubation (23). Therefore, except in experiments with normoglycemic hypoxia, spinal roots in this study were preincubated for $3-6 \mathrm{~h}$ in different hexose-containing solutions. These incubation media contained $2.5 \mathrm{mM}$ D-glucose plus $22.5 \mathrm{mM}$ of $\mathrm{D}$-galactose, L-glucose, D-fructose, or L-fucose. This protocol enabled us to record consecutively from several spinal roots in a given experiment.

Solutions. The standard bicarbonate-free solution used contained $118.0 \mathrm{mM} \mathrm{NaCl}, 20.0 \mathrm{mM}$ sodium gluconate, $3.0 \mathrm{mM} \mathrm{KCl}, 1.5 \mathrm{mM} \mathrm{CaCl}_{2}, 1.0 \mathrm{mM} \mathrm{MgCl}_{2}$, and $6.0 \mathrm{mM}$ HEPES. The concentration of $D$-glucose was either 2.5 or $25 \mathrm{mM}$. All other hexoses (L-glucose, D-mannose, D-galactose, D-fructose, L-fucose) (Sigma, Deisenhofen, Germany) were added in a concentration of $22.5 \mathrm{mM}$ to the standard solution containing $2.5 \mathrm{mM} \mathrm{D}$-glucose. lodoacetate ( $\mathrm{Na}^{+}$salt) (Sigma) was used in a concentration of $10 \mathrm{mM}$. The normoxic solution was equilibrated with $21 \%$ $\mathrm{O}_{2} / 79 \% \mathrm{~N}_{2}$ and the hypoxic solution with ultra pure argon. The $\mathrm{pO}_{2}$ in the organ bath was monitored contin- uously by a Clark-style electrode (Diamond Electro-Tech, Ann Arbor, MI) and found to be $<2 \mathrm{mmHg}$ within 1-2 min after perfusion of the organ bath with the hypoxic solutions. Ultra pure argon (Argon 6.0) was obtained from Linde (Unterschleißheim, Germany).

CNAP recordings. The organ bath used to record CNAPs and extracellular dc potentials has been described previously $(23,24)$. The bath consisted of a three-chambered Plexiglas bath compartmentalized by 1 $\mathrm{mm}$ partitions (Marsh ganglion bath; Hugo Sachs Elektronik, March-Hugstetten, Germany). Each partition had removable upper and lower sections in which a slot had been cut to allow the spinal roots to pass between chambers without being crushed. Silicone grease was used to seal the root into position and to prevent the free diffusion of solutions between chambers. The central compartment was perfused continuously by positive gas pressure in buffer flasks. The flow rate was $14 \mathrm{ml} / \mathrm{min}$ (volume of the central compartment was $1.5 \mathrm{ml}$ ); bath temperature was adjusted to $36^{\circ} \mathrm{C}$. The nerve end in one of the lateral compartments was drawn into a suction electrode that was used for stimulation (supramaximal single square voltage pulses: duration, $0.1 \mathrm{~ms}$; amplitude, $80-100 \mathrm{~V}$; stimulation rate, $0.2 \mathrm{~Hz}$ ). The solution in this lateral compartment was identical to the one in the central compartment; however, it was not made hypoxic. The $\mathrm{K}^{+}$concentration in the second lateral compartment was elevated by $30 \mathrm{mM}$. A pair of dc stable silver/silver chloride recording electrodes was used to record CNAPs (bandwith of preamplifier, 0.03-2 kHz; amplification, $\times 200)$ and the extracellular dc potential (dc, -2 $\mathrm{kHz}$; amplification, $\times 10$ ) across the resistance between the second lateral and the central compartment of the organ bath. The origin of the dc or demarcation potential has been discussed in detail by Lorente de Nó (21). This represents a reliable fraction of the membrane potential and was used because stable intra- axonal recordings for $\geq 1 \mathrm{~h}$ were not possible. A peak detector enabled us to measure continuously the height of each action potential peak.

pH-sensitive microelectrodes. Double-barreled $\mathrm{pH}$ sensitive twisted-type microelectrodes were constructed according to a method described previously by Ballanyi and Schlue (25). The central barrel contained the $\mathrm{pH}$ sensitive cocktail (Hydrogen lon lonophore I-Cocktail A 95291; Fluka, Buchs, Switzerland) and was backfilled with a mixed electrolyte solution of $100 \mathrm{mM} \mathrm{Na}^{+}$citrate and $100 \mathrm{mM} \mathrm{NaCl}$ (pH adjusted to 6.0). The reference barrel was filled with $1 \mathrm{~mol} / \mathrm{L} \mathrm{Mg}^{2+}$ acetate solution. Each electrode was calibrated in a HEPES-buffered $(6 \mathrm{mM})$ standard solution adjusted to $\mathrm{pH} 7.4$ and in a PIPESbuffered $(6 \mathrm{mM})$ standard solution adjusted to $\mathrm{pH} 6.4$. The average slope of the electrodes was $54.8 \mathrm{mV} / \mathrm{pH} U$ $(n=8)$. During the experiments, the double-barreled $\mathrm{pH}$-sensitive microelectrodes were inserted into the center of the spinal roots for continuous measurement of the $\mathrm{pH}_{\mathrm{e}}$.

Statistical analysis. Data were recorded, stored, and analyzed (averaged) on a digital oscilloscope (Nicolet 4562, Offenbach, Germany). Because the absolute values of the amplitude of the CNAP varied between differ- 
A

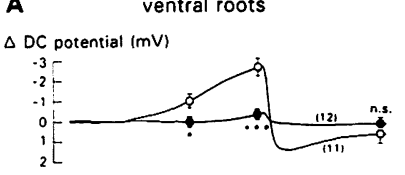

B

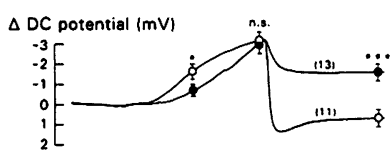

peak height $(\%)$

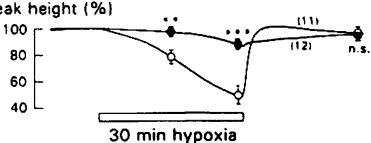

$02.5 \mathrm{mM}$ D.glucose

- $25 \mathrm{mM}$ D-glucose

peak height $(\%)$

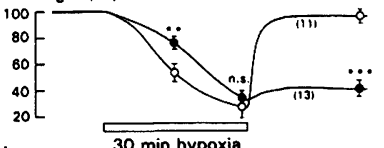
FIG. 1. Changes in the peak height of CNAP and extracellular dc
potential $(\triangle \mathrm{DC})$ before, during, and after hypoxia. $A$ : observations made on ventral spinal roots incubated in solutions containing either 2.5 or $25 \mathrm{mM}$ D-glucose. B: observations made on dorsal spinal roots incubated in the same concentrations of $D$-glucose. Data were digitized and averaged (means \pm SE, number of roots in parenthesis). Only one exposure to hypoxia was performed on each spinal root. " $P<0.05$; $" P<0.01 ; " * p<0.001$.

ent spinal roots, the digitized data were normalized to the same pre-hypoxic level. This enabled the data to be compared with the percentage changes in CNAP and to obtain unbiased averages of data from different groups. Averages of shifts in the extracellular dc potential were obtained by using changes in the reading of absolute potential compared with the normalized pre-hypoxic level. Data are expressed as means \pm SE. Statistical analysis was performed by an unpaired two-tailed Student's $t$ test to assess significance of differences.

\section{RESULTS}

Resistance to hypoxia and liability to hypoxia-related electrophysiological damage have been observed previously in isolated peripheral nerves exposed to hyperglycemic hypoxia (21-23). The following experiments were designed in view of two observations made in these studies. First, resistance to hypoxia was more prominent in motor compared with sensory nerve fibers (23). Accordingly, in this study, the effects of different hexoses were separately tested on ventral and dorsal spinal roots. Second, the $\mathrm{pH}$-buffering power of the bathing solution and/or $\mathrm{HCO}_{3}{ }^{-}$-dependent $\mathrm{pH}$ regulation mechanisms determined posthypoxic functional recovery $(22,23)$. Therefore, with the aim to study the mechanisms underlying hyperglycemic functional damage, all experiments in this study were performed in bicarbonate-free solutions (see DISCUSSION).

Figure 1 illustrates the effects of hypoxia on extracellular dc and CNAP of isolated rat spinal roots incubated in solutions with normal $(2.5 \mathrm{mM})$ and high $(25 \mathrm{mM})$ concentrations of D-glucose. Resistance to hypoxia is revealed clearly on ventral roots. Both membrane depolarization and the decline in the amplitude of the CNAP were reduced strongly when the ventral spinal roots were exposed to hypoxia in solutions containing $25 \mathrm{mM} \mathrm{D}$-glucose. The electrophysiological damage caused by hyperglycemic hypoxia, on the other hand, is revealed in experiments on dorsal spinal roots. Neither membrane potential nor action potential amplitude recovered after hypoxia in solutions containing $25 \mathrm{mM}$ D-glucose. Thus,

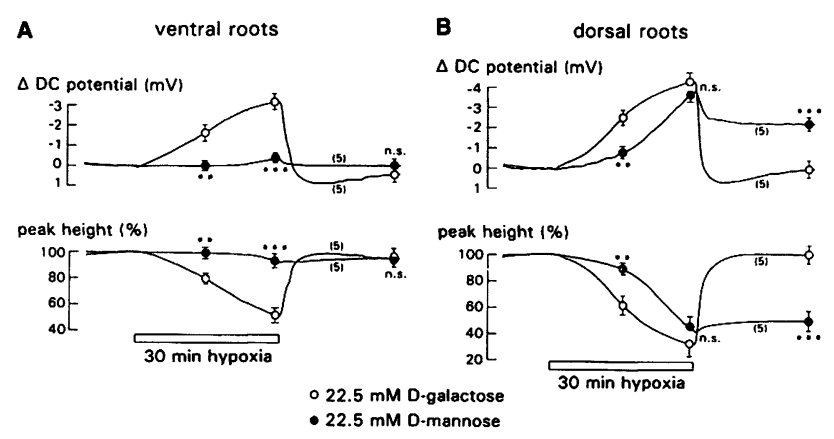

FIG. 2. Changes in the peak height of CNAP and extracellular dc potential $(\triangle \mathrm{DC})$ before, during, and after hypoxia. $A$ : observations made on ventral spinal roots incubated for several hours before and during hypoxia in solutions containing either $2.5 \mathrm{mM} \mathrm{D-glucose}$ plus $22.5 \mathrm{mM}$ D-mannose or $2.5 \mathrm{mM}$ D-glucose plus $22.5 \mathrm{mM}$ D-galactose. $B$ : observations made on dorsal spinal roots incubated in solutions containing the same concentrations of hexoses. Data were digitized and averaged (means \pm SE, number of roots in parentheses). Only one exposure to hypoxia was performed on each spinal root. " $P<0.01$; P $<0.001$.

clear evidence exists for functional impairment of spinal root axons.

Based on these observations, we explored whether high concentrations of other hexoses would also result in resistance to hypoxia and/or liability to hypoxic electrophysiological damage. Spinal roots were first incubated for 3-6 $\mathrm{h}$ in solutions containing $2.5 \mathrm{mM}$-glucose plus $22.5 \mathrm{mM}$ of other hexoses. Afterwards, such spinal roots were exposed to hypoxia for $30 \mathrm{~min}$. We observed that only the nerves incubated in high concentrations of D-mannose showed resistance to hypoxia and failed to recover functionally from hypoxia; D-galactose, D-fructose, L-fucose and L-glucose did not produce such effects. These findings are illustrated in Figs. 2 and 3.

Figure 2 shows averaged, original recordings from 10 ventral and 10 dorsal spinal roots that had been incubated in either $2.5 \mathrm{mM} \mathrm{D}$-glucose plus $22.5 \mathrm{mM}$ D-mannose or in $2.5 \mathrm{mM}$ D-glucose plus $22.5 \mathrm{mM}$ D-galactose. We tested the effects of hypoxia on the extracellular dc potential and on the peak height of CNAP. Only one exposure to hypoxia was performed on each spinal root. A clear difference existed in the effects of hypoxia between spinal roots incubated in high concentrations of D-mannose compared with those incubated in high concentrations of $\mathrm{D}$-galactose. The former revealed resistance to hypoxia (Fig. $2 A$ ) and impairment of posthypoxic functional recovery (Fig. $2 B$ ); the latter failed to show such alterations in their response to hypoxia. Similar experiments were performed on other spinal roots that had been incubated in high concentrations of L-glucose, D-fructose, and L-fucose before and during exposure to hypoxia. These hexoses, too, did not induce a change in the sensitivity to hypoxia compared with spinal roots exposed to hypoxia in the standard solution with $2.5 \mathrm{mM}$ D-glucose (Fig. 3). Thereby, changes in the peak height of CNAPs taken (from ventral roots) at the end of exposure to hypoxia (30 min) and (from dorsal roots) $20 \mathrm{~min}$ after recovery to normal oxygenation are compared. It became evident that high concentrations of $D$-mannose imitate the effects of $25 \mathrm{mM} \mathrm{D}$-glucose. All other hexoses 
peak height

A

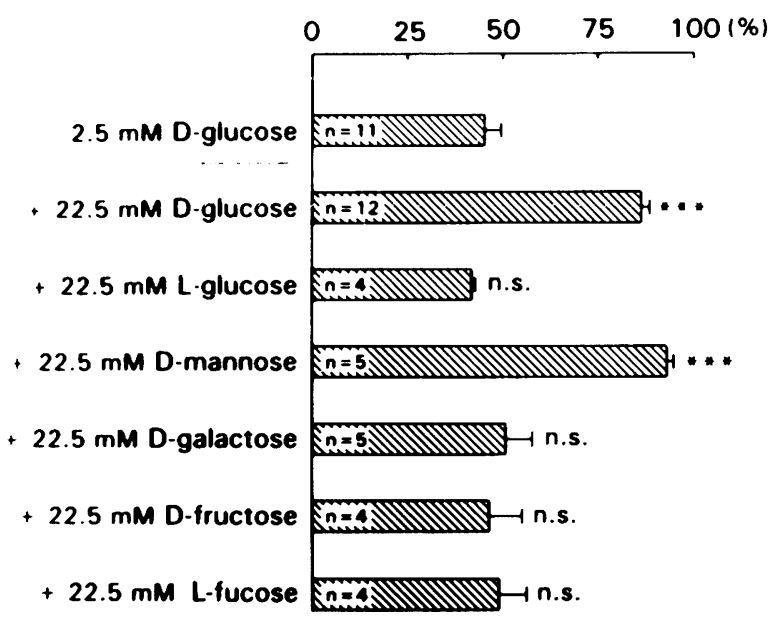

ventral roots

hypoxia $(30 \mathrm{~min})$
B

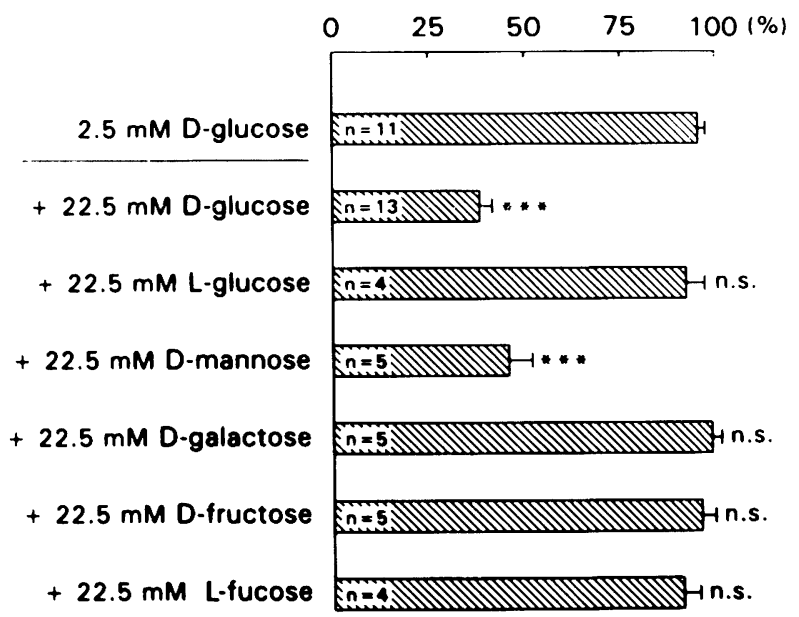

FIG. 3. Summary of the effects of different hexoses on the sensitivity of spinal roots to hypoxia. A: peak heights of CNAP in ventral spinal roots after 30 min of exposure to hypoxia. B: peak heights of CNAP in dorsal spinal roots 20 min after end of exposure to hypoxia for 30 min (means \pm SE; $n$, roots; $100 \%$, peak height before hypoxia). Only one exposure to hypoxia was performed on each spinal root. "'P $<0.001$.

tested did not modify the sensitivity of the spinal roots to hypoxia compared with spinal roots incubated in the standard solution with $2.5 \mathrm{mM}$ D-glucose.

In another series of experiments, spinal roots incubated in $25 \mathrm{mM}$ D-glucose were simultaneously exposed to both hypoxia and inhibition of glycolysis (Fig. 4). Glycolysis was inhibited by $10 \mathrm{mM}$ iodoacetate, an inhibitor of the enzyme phosphoglyceraldehyde dehydrogenase. Exposure of spinal roots to iodoacetate (10 $\mathrm{mM}$ ) for 30 min during normal oxygenation did not result in axonal membrane depolarization or a decrease in the peak height of the CNAPs (observations on three ventral and four dorsal spinal roots; not shown). In contrast, iodoacetate strongly enhanced the effects of hypoxia.

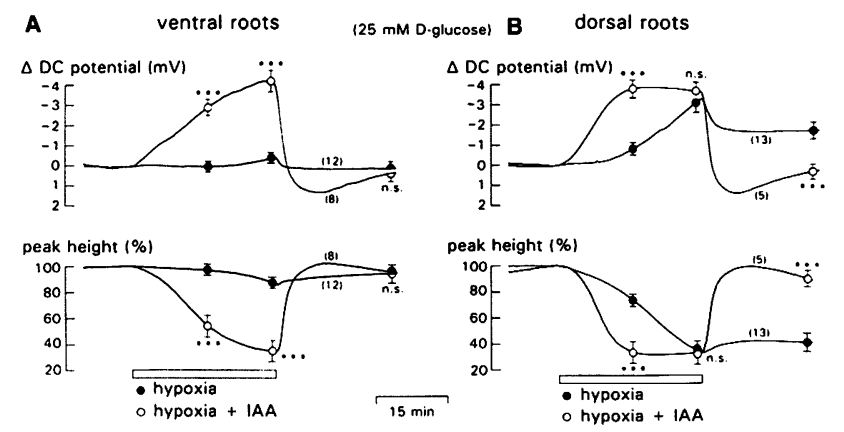

FIG. 4. Changes in the peak height of CNAP and extracellular dc potential $(\Delta D C)$ before, during, and after hypoxia. $A$ : observations made on ventral spinal roots incubated for several hours before and during hypoxia in a solution containing $25 \mathrm{mM}$ D-glucose. Such roots were exposed to either hypoxia or to both hypoxia and inhibition of glycolysis by means of lodoacetate $(I A A, 10 \mathrm{mM})$. $B$ : the same type of experiment performed on dorsal spinal roots. Data were digitized and averaged (means $\pm S E$, number of roots in parentheses). Only one exposure to hypoxia was performed on each spinal root. "*tp $<0.001$.
Action potential amplitude and extracellular dc potential of both ventral and dorsal roots responded very sensitively to hypoxia, although the spinal roots had been incubated in $25 \mathrm{mM}$ D-glucose for several hours. Furthermore, iodoacetate improved posthypoxic functional recovery in spite of the augmented response during hypoxia. On average, peak height of dorsal spinal roots incubated in $25 \mathrm{mM}$ D-glucose recovered to only $38.2 \pm 3.3 \%(n=13)$ of the prehypoxic level; however, the recovery was nearly complete $(88.6 \pm 3.4 \% ; n=5)$ when hypoxia was induced with simultaneous inhibition of glycolysis (Fig. 4).

Experiments with $\mathrm{pH}$-sensitive microelectrodes revealed a clear correlation between glucose availability and changes in $\mathrm{pH}_{e}$ (Fig. 5). Figure $5 A$ shows recordings made with $\mathrm{pH}$-sensitive microelectrodes that had been positioned to record $\mathrm{pH}_{\mathrm{e}}$ in the center of ventral spinal roots. Normoglycemic hypoxia induced an interstitial acidification of $0.21 \pm 0.03 \mathrm{pH} \cup$ (means $\pm \mathrm{SE}, n=6$ ). Hyperglycemic hypoxia (25 mM D-glucose), on the other hand, significantly enhanced the hypoxia-induced acidification to $0.33 \pm 0.04 \mathrm{pH} \cup(n=6, P<0.01)$. The most striking finding, however, was the almost complete $a b$ sence of an acid-going shift in $\mathrm{pH}_{\mathrm{e}}$ when hyperglycemic hypoxia was induced in the presence of iodoacetate (10 $\mathrm{mM}$ ). A small acidification of only $0.03 \pm 0.02 \mathrm{pH} \mathrm{U}$ $(n=4)$ was observed under these circumstances. The transient shifts in $\mathrm{pH}_{\mathrm{e}}$ observed at the start and end of iodoacetate application are to be expected during transmembranal diffusion of a weak acid (26). Recordings of $\mathrm{pH}_{\mathrm{e}}$ in dorsal spinal roots revealed a quantitatively similar relationship between glucose availability and hypoxiainduced interstitial acidification (Fig. 5B). 


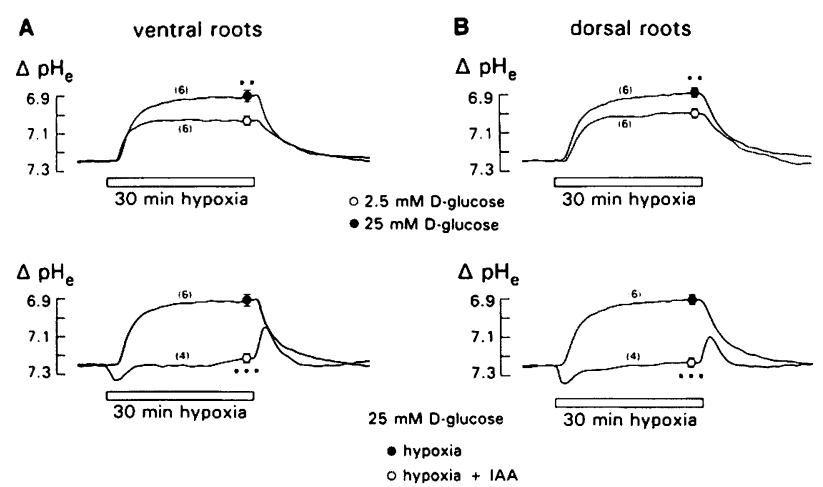

FIG. 5. Changes in $\mathrm{pH}_{6}$ recorded with $\mathrm{pH}$-sensitive microelectrodes positioned in the center of isolated rat spinal roots. $A$ : observations made on ventral spinal roots incubated in solutions containing either 2.5 or $25 \mathrm{mM}$ D-glucose. The hyperglycemic spinal roots were exposed to either hypoxia or hypoxia plus iodoacetate (IAA, $10 \mathrm{mM}$ ). B: similar experiments made on dorsal spinal roots. Data were digitized and averaged (means $\pm S E$, number of roots in parentheses). Only one exposure to hypoxia was performed on each spinal root. " $P<0.01$; ...p $<0.001$.

\section{DISCUSSION}

Two main findings have been obtained in this study. First, high concentrations of D-mannose alter the sensitivity of peripheral nerves to hypoxia in a manner similar to that shown for high concentrations of D-glucose. In contrast, L-glucose, D-galactose, L-fucose, and D-fructose failed to produce such an effect. Second, resistance to hypoxia, hypoxia-induced electrophysiological damage, and hypoxia-related interstitial acidification were completely absent in hyperglycemic nerves in which glycolysis had been inhibited by iodoacetate. The following conclusions can be drawn from these observations concerning two possible mechanisms underlying the abnormal response of hyperglycemic nerves to hypoxia.

Accumulation of polyols. Accumulation of osmotically active polyols such as sorbitol, galactitol, and mannitol may contribute to hypoxia-induced electrophysiological damage of spinal roots. However, in contrast to glucose and mannose, incubation of spinal roots for $3-6 \mathrm{~h}$ in 22.5 $\mathrm{mM}$ galactose did not inhibit functional recovery after hypoxia. Even isotonic galactose failed to produce such an effect in isolated frog sciatic nerve (21). Glucose, galactose, and mannose are all known to produce polyol accumulation in sciatic nerve and cultured neuroblastoma cells (27-29). Differences in the effects on posthypoxic functional recovery indicate, therefore, that accumulation of polyols seems not to contribute importantly to the electrophysiological damage seen in this study after hyperglycemic hypoxia.

Anaerobic glycolysis. Another possibility underlying the abnormal sensitivity to hypoxia of hyperglycemic nerves is enhanced glycolysis, as suggested previously (8-12). The possible importance of this mechanism is strongly supported by the effects of $D$-mannose seen in this study. It is known that D-mannose is converted to mannose-6 phosphate by hexokinase as effectively as the phosphorylation of glucose (30). By means of phosphomannoseisomerase, which is active in nervous tissue, mannose can enter directly into the glycolytic pathway and replace glucose (31). In our experiments, high concentrations of D-mannose imitated all of the effects of high concentrations of D-glucose. This observation is a strong argument that both resistance to hypoxia and liability to hypoxic damage are related to enhanced anaerobic glycolysis. These data are in apparent conflict with Calcutt et al. (32) and Lindström et al. (33), who showed that resistance to hypoxia is not produced in normal nerves by raising ambient glucose levels to those found in diabetic plasma. However, those researchers studied acute effects, whereas we have shown that nerves have to be incubated for several hours in high glucose concentrations for induction of an altered sensitivity to hypoxia (23). Also, the very thin external sheath of spinal roots seems to be better suited for diffusion of glucose from the bathing solution into the nerve compared with a multifascicular peripheral nerve with many perineurial barriers such as the sciatic nerve.

Further, strong support for the possible importance of enhanced anaerobic glycolysis comes from our finding that resistance to hypoxia and posthypoxic electrophysiological damage were absent after pharmacological inhibition of nerve glycolysis with iodoacetate. However, posthypoxic functional recovery seems unlikely when inhibition of glycolysis results in a lack of substrate for oxidative metabolism. The fact that CNAPs and membrane potential recovered after hypoxia in the presence of iodoacetate indicates that the concentration of the inhibitor used $(10 \mathrm{mM})$ and the time of application (30 min) did not completely block glycolysis. Spinal roots with a low rate of glycolysis such as those incubated in $2.5 \mathrm{mM} \mathrm{D}$-glucose, on the other hand, show complete functional posthypoxic recovery.

Mechanism of posthypoxic electrophysiological damage. A bathing solution of low buffering power $(6 \mathrm{mM}$ HEPES) was used in this study to reveal electrophysiological damage after hyperglycemic hypoxia. In contrast, we have shown previously that spinal roots in a bathing solution containing $25 \mathrm{mM} \mathrm{HCO}_{3}{ }^{-}$do not show a lack of functional posthypoxic recovery (23). However, to imitate ischemia in an in vitro experiment, a combination of hypoxia plus low buffering power is not unreasonable. In fact, it is known in the CNS that tissue $\mathrm{HCO}_{3}{ }^{-}$is exhausted during hyperglycemic ischemia (34). Under these circumstances, tissue acidosis as a result of enhanced anaerobic glycolysis seems to be the most important factor for hypoxia-induced electrophysiological damage seen in hyperglycemic spinal roots. This conclusion is strongly supported by the observations made in the presence of iodoacetate. This inhibitor of glycolysis blocked hypoxia-induced interstitial acidification and, in the same hyperglycemic spinal roots, improved posthypoxic functional recovery. The exact link between acidosis and neuronal damage is not yet clear. Secondary effects of low pH might be alterations in the behavior of ion channels and transporters, intracellular calcium accumulation, impaired neuronal energy metabolism, and/or disturbed cell volume regulation $(35,36)$.

Other views exist, however, with regard to the consequences of high glucose concentrations for posthypoxic functional recovery. Optic nerves, in contrast to our 
observations on spinal roots, showed significantly less damage after $60 \mathrm{~min}$ of anoxia in the presence of $20 \mathrm{mM}$ glucose compared with $10 \mathrm{mM}$ glucose (37). However, the experiments on optic nerves were performed in a bathing solution containing $26 \mathrm{mM} \mathrm{HCO}_{3}{ }^{-}$. This fact may explain the apparent discrepancy, because, as discussed above, tissue buffering power and/or $\mathrm{HCO}_{3}{ }^{-}$dependent $\mathrm{pH}$ regulation mechanisms contribute importantly to posthypoxic functional recovery. Hypoxia in high $\mathrm{HCO}_{3}{ }^{-}$concentrations does not imitate the in vivo situation when $\mathrm{HCO}_{3}{ }^{-}$stores are exhausted by hyperglycemic ischemia (34).

Other possible mechanisms underlying ischemic damage of peripheral nerves have been discussed recently (38). Thereby, factors such as production of oxygen free radicals, a massive rise in cytosolic calcium concentration, and/or alterations in lipid metabolism have been considered. The data obtained in this study indicate that, in particular for hyperglycemic nerve ischemia, tissue acidosis should be added to this list of possible mechanisms.

Relevance to diabetic neuropathy. These data reveal a hazardous situation for diabetic patients. On one hand, resistance of their peripheral nerves to ischemia results in a decreased sensation of nerve hypoxia. Therefore, in contrast to normoglycemic subjects, nerves of diabetic patients may be exposed much longer to ischemia. On the other hand, under such circumstances, axonal acidosis may reach dangerous levels as a result of a long-lasting activation of anaerobic glycolysis and to a decrease in the capability of the nerve to regulate $\mathrm{pH}$ caused by a use-dependent $\mathrm{HCO}_{3}{ }^{-}$decrease. Very likely, such a mechanism contributes to the liability of diabetic nerves to hypoxic damage. However, it may well contribute to the development of diabetic neuropathy in general.

\section{ACKNOWLEDGMENTS}

This work was supported by the German Research Foundation (SFB 220/B1).

We would like to thank $C$. Müller for expert technical and secretarial assistance.

\section{REFERENCES}

1. Low PA: Recent advances in the pathogenesis of diabetic neuropathy. Muscle Nerve 10:121-28, 1987

2. Thomas PK, Tomlinson DR: Diabetic and hypoglycemic neuropathy. In Peripheral Neuropathy. Vol. 2. Dyck PJ, Thomas PK, Griffin JW, Low PA, Poduslo JF, Eds. Philadelphia, PA, Saunders, 1993, p. $1219-50$

3. Steiness I: Vibratory perception in diabetics during arrested blood flow to the limb. Acta Med Scand 163:195-205, 1959

4. Thomas PK, Brown MJ: Diabetic polyneuropathy. In Diabetic Neuropathy. Dyck PJ, Thomas PK, Asbury AK, Winegrad Al, Porte D, Eds. Philadelphia, Saunders, 1987, p. 56-65

5. Seneviratne KN, Peiris OA: The effects of hypoxia on the excitability of the isolated peripheral nerves of alloxan-diabetic rats. J Neurol Neurosurg Psychiatry 32:462-69, 1969

6. Jakobsen J: Peripheral nerves in early experimental diabetes: expansion of the endoneurial space as a cause of increased water content. Diabetologia 14:113-19, 1978

7. Christensen $\mathrm{JN}$, Orskov $\mathrm{H}$ : Vibratory perception during ischemia in uremic patients and in subjects with mild carbohydrate intolerance (Abstract). J Neurol Neurosurg Psychiatry 32:519, 1969

8. Low PA, Ward K, Schmelzer JD, Brimijoin S: Ischemic conduction failure and energy metabolism in experimental diabetic neuropathy. Am J Physiol 248:E457-62, 1985
9. Jaramillo J, Simard-Duquesne N, Dvornik D: Resistance of the diabetic rat nerve to ischemic inactivation. Can J Physiol Pharmacol 63:773-77, 1985

10. Shirabe S, Kinoshita I, Matsuo $H$, Takashima $H$, Nakamura $T$, Tsujihata M, Nagataki S: Resistance to ischemic conduction block of the peripheral nerve in hyperglycemic rats: an electrophysiological study. Muscle Nerve 11:582-87, 1988

11. Parry GJ, Kohzu H: Studies of resistance to ischemic nerve conduction failure in normal and diabetic rats. J Neurol Sci 93:61-67. 1989

12. Strupp $M$, Bostock $H$, Weigl $P$, Piwernetz $K$, Renner R, Grafe $P$ : Is resistance to ischaemia of motor axons in diabetic subjects due to membrane depolarization? J Neurol Sci 99:271-80, 1990

13. Greene DA, Lattimer SA, Sima AAF: Are disturbances of sorbitol, phosphoinositide, and $\mathrm{Na}+\mathrm{K}+\mathrm{ATPase}$ regulation involved in pathogenesis of diabetic neuropathy? Diabetes 37:688-93, 1988

14. Low PA, Schmelzer JD, Ward KK, Yao JK: Experimental chronic hypoxic neuropathy: relevance to diabetic neuropathy. Am J Physiol 250:E94-99, 1986

15. Hampton KK, Alani SM, Wilson Jl, Price DE: Resistance to ischaemic conduction failure in chronic hypoxaemia and diabetes. $J$ Neurol Neurosurg Psychiatry 52:1303-305, 1989

16. Cameron NE, Cotter MA, Robertson S: Angiotensin converting enzyme inhibition prevents development of muscle and nerve dysfunction and stimulates angiogenesis in streptozotocin-diabetic rats. Diabetologia 35:12-18, 1992

17. Asbury AK. Focal and multifocal neuropathies of diabetes. In Diabetic Neuropathy. Dyck PJ, Thomas PK, Asbury AK, Winegrad Al, Porte D, Eds. Philadelphia, Saunders, 1987, p. 45-55

18. Baba, M. Diabetic nerve function: its electrophysiological evaluation. In Diabetic Neuropathy. Ward J, Goto Y, Eds. Chichester, UK, Wiley, 1990 , p. 269-80

19. Ram Z, Sadeh M, Walden R, Adar R: Vascular insufficiency quantitatively aggravates diabetic neuropathy. Arch Neurol 48:1239-42. 1991

20. Nukada $\mathrm{H}$ : Mild ischemia causes severe pathological changes in experimental diabetic nerve. Muscle Nerve 15:1116-22, 1992

21. Lorente de No, R. A Study of Nerve Physiology. New York, Rockefeller Institute for Medical Research, 1947

22. Strupp $M$, Jund $R$, Schneider $U$, Grafe $P$ : Glucose availability and sensitivity to anoxia of isolated rat peroneal nerve. Am J Physiol 261:E389-94, 1991

23. Schneider $U$, Jund $R$, Nees $S$, Grafe $P$ : Differences in sensitivity to hyperglycemic hypoxia of isolated rat sensory and motor nerve fibers. Ann Neurol 31:605-10, 1992

24. Marsh SJ, Stansfeld CE, Brown DA, Davey R, McCarthy D: The mechanism of action of capsaicin on sensory $C$-type neurons and their axons in vitro. Neuroscience 23:275-89, 1987

25. Ballanyi $\mathrm{K}$, Schlue WR: Electrophysiological characterization of a nicotinic acetylcholine receptor on leech neuropile glial cells. Glia 2:330-45, 1989

26. Marrannes $\mathrm{R}$, de Hemptinne $A$, Leusen $\mathrm{I}$ : $\mathrm{pH}$ aspects of transient changes in conduction velocity in isolated heart fibers after partial replacement of chloride with organic acids. Pflügers Arch 389:199209, 1981

27. Sharma AK, Thomas PK, Baker RWR: Peripheral nerve abnormalities related to galactose administration in rats. J Neurol Neurosurg Psychiatry 39:794-802, 1976

28. Willars GB, Lambourne JE, Tomlinson DR: Does galactose feeding provide a valid model of consequences of exaggerated polyolpathway flux in peripheral nerve in experimental diabetes? Diabetes $36: 1425-31,1987$

29. Yorek MA, Dunlap JA, Leeney EM: Effect of galactose and glucose levels and sorbinil treatment on myo-inositol metabolism and $\mathrm{Na}+/ \mathrm{K}+$ pump activity in cultured neuroblastoma cells. Diabetes 38:996-1004, 1989

30. Sokoloff L: Circulation and energy metabolism of the brain. In Basic Neurochemistry. Siegel G, Agranoff B, Albers RW, Molinoff P, Eds. New York, Raven, 1989, p. 565-90

31. Sloviter HA, Kamimoto T: The isolated, perfused rat brain preparation metabolizes mannose but not maltose. J Neurochem 17:110911,1970

32. Calcutt NA, Ettlinger CB, Carrington $A L$, Diemel $L$, Tomlinson DR: Resistance to hypoxic conduction block in sciatic nerves of rats with streptozotocin-induced diabetes mellitus. J Neurol Sci 103:116-23, 1991

33. Lindström $P$, Brismar $T$ : Mechanism of anoxic conduction block in mammalian nerve. Acta Physiol Scand 141:429-33, 1991

34. Kraig RP, Pulsinelli WA, Plum F: Carbonic acid buffer changes during complete brain ischemia. Am J Physiol 250:R348-57. 1986

35. Siesjö BK: Acidosis and ischemic brain damage. Neurochem Pathol 
9:31-88, 1988

36. Sutherland GR, Peeling J, Sutherland E, Tyson R, Dai F, Kozlowski $P$, Saunders JK: Forebrain ischemia in diabetic and nondiabetic BB rats studied with ${ }^{31} \mathrm{P}$ magnetic resonance spectroscopy. Diabetes 41:1328-34, 1992

37. Ransom BR, Walz W, Davis PK, Carlini WG: Anoxia-induced changes in extracellular $\mathrm{K}^{+}$and $\mathrm{pH}$ in mammalian central white matter. J Cereb Blood Flow Metab 12:593-602, 1992

38. McManis PG, Low PA, Lagerlund TG: Microenviroment of nerve, blood flow and ischemia. In Peripheral Neuropathy. Vol. 2. Dyck PJ Thomas PK, Griffin JW, Low PA, Poduslo JF, Eds. Philadelphia, Saunders, 1993, p. 453-73 